\title{
A Hybrid the Nonsubsampled Contourlet Transform and Homomorphic Filtering for Enhancing Mammograms
}

\author{
Khaddouj Taifi*, Rachid Ahdid, Mohamed Fakir, Said Safi \\ Faculty of Science and Technology Beni-Mellal, Morocco \\ Laboratory of Information Processing \& Decision Support (TIAD) \\ Faculty of Science and Technics, University Sultan Moulay Slimane \\ ${ }^{*}$ Corresponding author, e-mail: taif_kha@hotmail.fr
}

\begin{abstract}
Mammogram is important for early breast cancer detection. But due to the low contrast of microcalcifications and noise, it is difficult to detect microcalcification. This paper presents a comparative study in digital mammography image enhancement based on three different algorithms: homomorphic filtering, unsharp masking and our proposed methods. This latter uses a hybrid method Combining contourlet and homomorphic filtering. Performance of the given technique has been measured in terms of distribution separation measure (DSM), target-tobackground enhancement measure based on standard deviation (TBES) and target-to-background enhancement measure based on entropy (TBEE). The proposed methods were tested with the referents mammography data Base MiniMIAS. Experimental results show that the proposed method improves the visibility of microcalcification.
\end{abstract}

Keyword: microcalcification, contourlet, enhancement homomorphic filtering

Copyright $@ 2015$ Institute of Advanced Engineering and Science. All rights reserved.

\section{Introduction}

Breast cancer is the most common cancer in women and ranks first in the world continues to be the leading cause of death over 40 years [1]. In Morocco, breast cancer is also the first woman and according to data from the register of the Greater Casablanca 2004 the incidence standardized on the world population is about 35 cases / 100,000 women /year it represents $36 \%$ of all women's cancers. According to hospital data and 2008 data, the incidence was 36.5 cases / 100,000 women / year [2].

Various studies have confirmed this is the detection of early stage breast cancer may improve prognosis. Mammography technique remains the essential detecting breast, the most efficient in monitoring and early detection of breast cancer. It helps to highlight potential radiological signs such as suspicious opacities which can translate from malignant lesions.

However, despite significant progress in terms of equipment, all radiologists recognize the difficulty of interpreting mammograms which further increased by the type of breast tissue examined.

Mammographic images show a contrast between the two main constituents of the breast fatty tissue and connective-fibrous matrix. In general, it is extremely difficult to define normality of mammographic images: Indeed, the appearance of the mammary gland is extremely variable depending on the patient's age and the period during which the mammogram is done.

Contrast enhancement has an important role in image processing as it extract the useful information from the distorted image. Image enhancement is used for improving the visual quality of an image. Objective of Image enhancement is to process an image so that result is more suitable than original image for specific application. Digital image enhancement techniques provide a multitude of choices for improving the visual quality of images.

The fundamental enhancement needed in mammography is an increase in contrast, especially for dense breasts. Contrast between malignant tissue and normal dense tissue may be present on a mammogram, but below the threshold of human perception. 
To illustrate edges and small details in a mammogram image, Unsharp masking filter is very useful [3].The unsharp masking method reduces low-frequency details but amplifies highfrequency.

This paper will provide an overview of contrast enhancement techniques it is organize as follows: Section 2, deals with contrast enhancement. Section 3, describes evaluation of contrast enhancement techniques for mammographic. Section 4, experimental results.

\section{Contrast Enhancement}

\subsection{Homomorphic Filtering}

Classical technique of histogram equalization does not produce an effective result in the presence of non-uniform illumination pattern in an image. Therefore it is necessary to develop a frequency domain approach that improves the appearance of an image by simultaneous graylevel range compression and contrast enhancement. A homomorphic frequency domain filtering technique is investigated in the case of various different illumination patterns in grey scale image as well as color image.

The homomorphic filter function decreases the energy of low frequencies and increases those of high frequencies in the image [4].

We can model an image (matrix of luminance) as the product of two characteristics: the first, called enlightenment and rated $0<i(x, y)<\infty$ is the amount of light incident on the scene view. The second, called reflectance and rated $0<r(x, y)<1$, is the amount of light reflected from objects in the scene, these two characteristic make up the overall perceived intensity, expressed as a product.

The illumination-reflectance model can be used to develop a frequency domain procedure for improving the appearance of an image by simultaneous gray-level range compression and contrast enhancement. An image $f(x, y)$ can be expressed as the product of illumination and reflectance components [5].

$$
f(x, y)=i(x, y) * r(x, y)
$$

Because the Fourier transform of the product of two functions is not separable, we define:

$$
z(x, y)=\ln f(x, y)=\ln i(x, y)+\ln r(x, y)
$$

Then Equation (2) became by using FT.

$$
\mathrm{z}(\mathrm{u}, \mathrm{v})=\mathrm{F}_{\mathrm{I}}(\mathrm{u}, \mathrm{v})+\mathrm{F}_{\mathrm{r}}(\mathrm{u}, \mathrm{v})
$$

Where $\mathrm{F}_{\mathrm{I}}(\mathrm{u}, \mathrm{v})$ and $\mathrm{F}_{\mathrm{r}}(\mathrm{u}, \mathrm{v})$ are the Fourier transforms of $\ln \mathrm{i}(\mathrm{x}, \mathrm{y})$ and $\ln \mathrm{r}(\mathrm{x}, \mathrm{y})$ respectively.

If we process $\mathrm{z}(\mathrm{u}, \mathrm{v})$ by means of a filter function $\mathrm{H}(\mathrm{u}, \mathrm{v})$, then, we obtain:

$$
\begin{aligned}
S(u, v) & =H(u, v) z(u, v) \\
& =H(u, v) F_{I}(u, v)+H(u, v) F_{r}(u, v)
\end{aligned}
$$

Where $\mathrm{S}(\mathrm{u}, \mathrm{v})$ is the Fourier transform of the result. In the spatial domain,

$$
\begin{aligned}
\mathrm{s}(\mathrm{x}, \mathrm{y}) & =\operatorname{IFFT}\{\mathrm{S}(\mathrm{u}, \mathrm{v})\} \\
& =\operatorname{IFFT}\left\{\mathrm{H}(\mathrm{u}, \mathrm{v}) \mathrm{F}_{\mathrm{I}}(\mathrm{u}, \mathrm{v})\right\}+\operatorname{IFFT}\left\{\mathrm{H}(\mathrm{u}, \mathrm{v}) \mathrm{F}_{\mathrm{r}}(\mathrm{u}, \mathrm{v})\right\}
\end{aligned}
$$

Where IFFT is the Inverse Fourier Transform.

So the output image can be expressed by the function:

$$
g(x, y)=e^{s(x, y)}
$$

The filter function of homomorphic systems can be shown below.

$$
H(u, v)=\frac{1}{1+\left(\frac{D(u, v)}{D_{0}}\right)^{2 n}}, D(u, v)=\sqrt{u^{2}+v^{2}}
$$




\subsection{Nonsubsampled Contourlet Transformation}

NonsubSampled Contourlet Transform (NSCT) proposed by Cunha, et al [6,7], is a invariant version by translation of transform contourlets. The transformed into contourlets uses a Laplacian pyramid [8] for the multiscale decomposition, the NonSubsampled Directional Filter Bancs for directional decomposition. To ensure the translation invariance, NSCT is implemented using a pyramidal structure NonsubSampled and directional filterbanks NonsubSampled.

Figure 1 illustrates the principle of NSCT. These combine two successive stages of decomposition invariant by translation:

(1) NonSubsampled Pyramid( NSP) which provides multi-scale

(2) NonSubsampled Directional Filter Banks (NSDFB) allowing decomposition according different orientations) [9].

The result is a flexible image decomposition, multiscale, translation invariant and Multidirection expansion that has better directional frequency localization and a fast implementation. NSCT consists of two filter banks, i.e. the NonSubsampled Pyramid Filter Bank (NSPFB) and the NonSubsampled Directional Filter Bank (NSDFB) as shown in Figure 1(a), which split the 2-D frequency plane in the subbands illustrated in Figure 1(b).

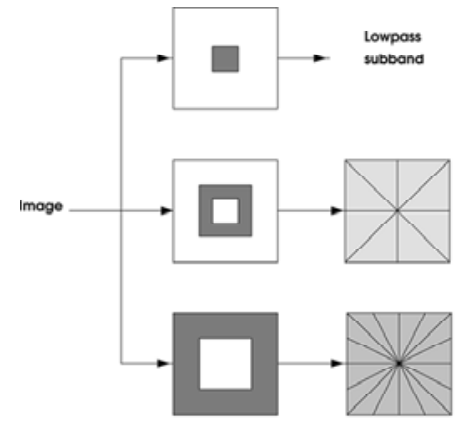

(a)

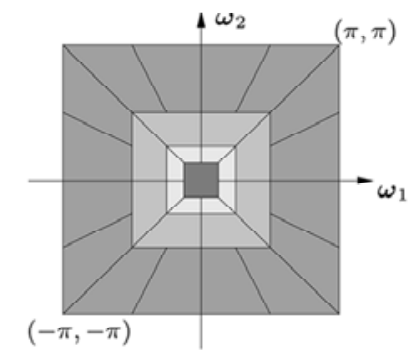

(b)

Figure 1. Nonsubsampled contourlet transform. (a) NSFB structure that implements the NSCT;

(b) Idealized frequency partitioning

Figure 2 presents a comparative study in digital mammography image enhancement based on three different algorithms: hommomorphique filter, unsharp masking and proposed methods (using a hybrid method Combining NonsubSampled Contourlet Transform and hommomorphique filter). Finally the enhanced image is obtained with clarity and free from noise.
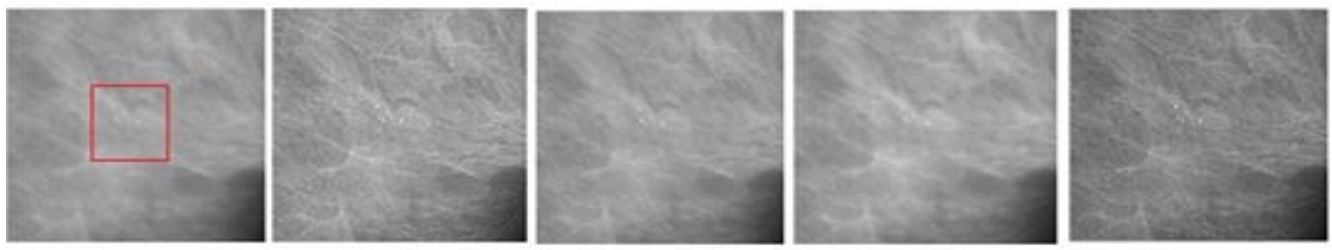

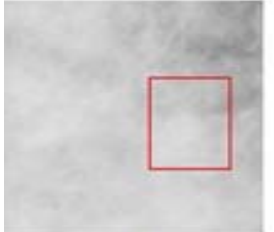

a
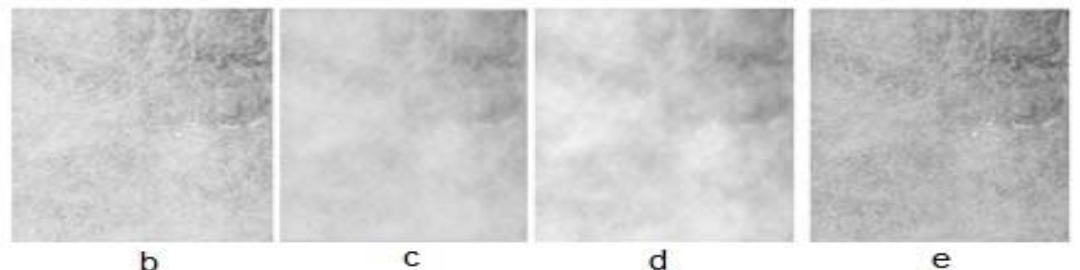

Figure 2. a) ROIs of the original image, b) the NSCT, c)the homomrphic filtering, d) the Unsharp masking,e) the proposed enhancement 
To verify the proposed method, experiments are performed with data base MIAS mammogram.

In the experiment, four contrast enhancement methods are performed b) the NSCT c)the homomrphic filtering and, d) the Unsharp masking and e) the proposed robust enhancement using modified homomorphic filter in contourlet (proposed enhancement). The proposed method estimates noise characteristic in background region and eliminates noise in breast area incorporation with contrast enhancement of microcalcification. Experimental results show that, the proposed enhancement significantly reduces noise in high noise mammograms.

\section{Evaluation of Contrast Enhancement Techniques for Mammographic}

To gauge the quality of the contourlet based enhanced image,three quantitative measures such as Distribution Separation Measure (DSM), Target to Back-ground contrast Enhancement measure based on Standard deviation (TBES), Target to Background contrast Enhancement measure based on Entropy (TBEE) S. Singh and K. Bovis proposed three different quantitative measures for evaluation of the enhanced image quality [10,11].

\subsection{Distribution Separation Measure (DSM)}

The DSM represents how separated are the distributions of each mammogram and is defined by Equation (8).

$$
\operatorname{DSM}=\left(\left|\mu_{\mathrm{T}}^{\mathrm{E}}-\mu_{\mathrm{mic}}^{\mathrm{E}}\right|\right)-\left(\left|\mu_{\mathrm{T}}^{\mathrm{O}}-\mu_{\mathrm{mic}}^{\mathrm{O}}\right|\right)
$$

Where, $\mu_{\mathrm{mic}}^{\mathrm{E}}, \mu_{\mathrm{mic}}^{\mathrm{O}}$ are the mean of the microcalcification region of the enhanced and original image respectively. $\mu_{\mathrm{T}}^{\mathrm{E}}, \mu_{\mathrm{T}}^{\mathrm{O}}$ are the mean of the surrounding tissue of the enhanced and original image respectively.

\subsection{Target-to-Background Contrast Enhancement Measure Based on Standard Deviation} (TBCS)

A key objective of a contrast enhancement is to maximize the difference between background and target mean gray level and ensure that the homogeneity of the mass is increased aiding the visualization of its boundaries and location. Using the ratio of the standard deviation of the grayscales within the target before and after the enhancement, we can quantify this improvement using the TBCS given in (9).

$$
\operatorname{TBCS}=\frac{\left(\frac{\mu_{T}^{\mathrm{E}}}{\mu_{\text {mic }}^{\mathrm{E}}}-\frac{\mu_{\mathrm{T}}^{\mathrm{O}}}{\mu_{\text {mic }}^{\mathrm{O}}}\right)}{\left(\frac{\sigma_{\mathrm{T}}}{\sigma_{\mathrm{T}}^{\mathrm{O}}}\right)}
$$

Where, $\sigma_{T}^{o}, \sigma_{T}^{E}$ are the standard deviation of the grayscales comprising the target and background before and after the enhancement. Assuming that the target has a smaller mean before and after enhancement compared to the background.

\subsection{Target-to-Background Contrast Enhancement Measure Based on Entropy (TBCE)}

This measure is an extension of the TBC metric.TBCE is based on the entropy of the regions rather than in the standard deviations and is defined by Equation (10).

$$
\mathrm{TBCE}=\frac{\left(\frac{\mu_{\mathrm{T}}^{\mathrm{E}}}{\mu_{\text {mic }}^{\mathrm{E}}}-\frac{\mu_{\mathrm{T}}^{\mathrm{O}}}{\mu_{\text {mic }}^{\mathrm{O}}}\right)}{\left(\frac{\varepsilon_{\mathrm{T}}}{\varepsilon_{\mathrm{T}}^{\mathrm{O}}}\right)}
$$

Where, $£_{T}^{E}$ and $£_{T}^{o}$,are the entropy of the target in the original and enhanced images. 


\section{Experimental Results}

Figure 3, Figure 4 and Figure 5 show the plots of DSM, TBCS and TBCE metrics respectively used to measure the enhancement ability of the NSCT, unsharp masking, hommomorphic filter and proposed method (proposedM)

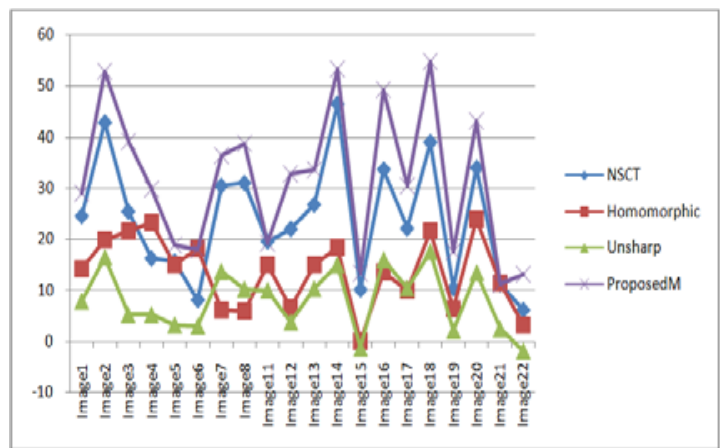

Figure 3. Plots of DSM metrics

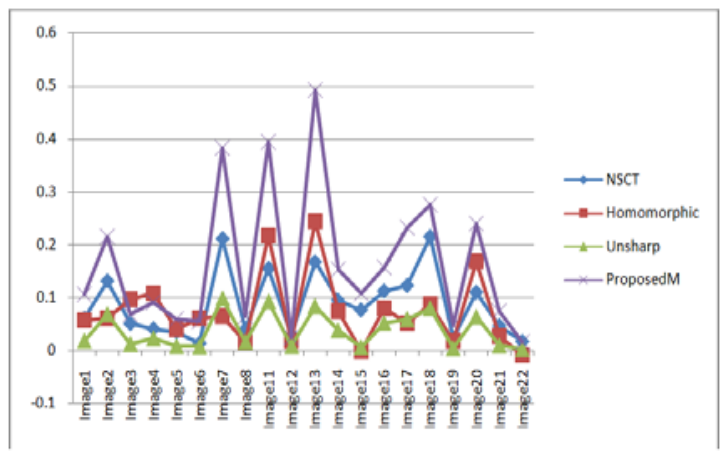

Figure 4. Plots of TBCS metrics

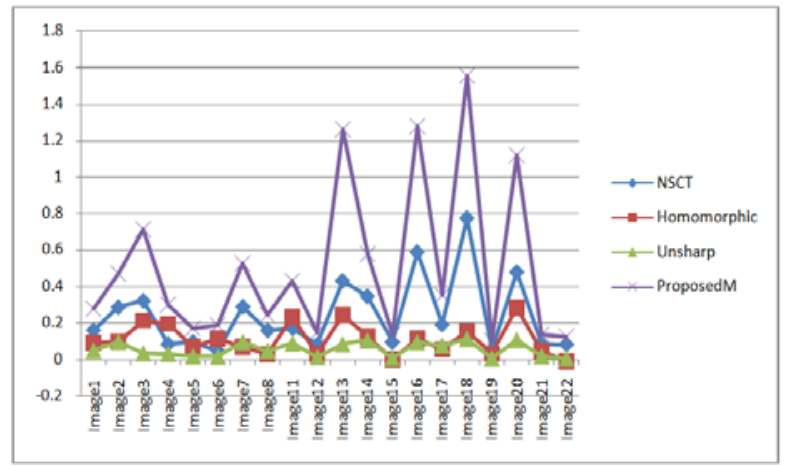

Figure 5. Plots of TBCE metrics

From these evaluation of contrast enhancement technique, we conclude that hybrid method (NSCT and homomorphic filter) gives good results for enhancing mammograms.

\subsection{Confusion Matrix}

To measure accuracy a medical test is done. Let's say we test some people for the presence of disease. Some of these people have the disease, and our test demonstrate that they are positive. They are called true positives (TP). Some have the disease, but the test says they do not. They are called false negatives (FN). Some do not have the disease, and the test said they do not - true negative (TN). Finally, there might be people in good health who have a positive result - false positives (FP). Thus, the number of true positives, false negatives, true negatives and false positives add up to $100 \%$ of the whole (Table 1).

Table 1. Confusion matrix

\begin{tabular}{llc}
\hline \multicolumn{1}{c}{ Actual } & Positive & Negative \\
\hline Positive & TP(True Positive) & FP(False Positive) \\
Negative & FN(False Negative) & TN(True Negative) \\
\hline
\end{tabular}

TP: correct classification of abnormal

FP: incorrect classification of abnormal

$\mathrm{TN}$ : correct classification of normal

$\mathrm{FN}$ : incorrect classification of normal 


\subsection{Database of Testing Set and Training Set Images}

A comparative study is done between wavelet and steerable pyramid transform to classify microcalcifications into normal or abnormal (Benign or Malignant) cases using multiorintation and multiresolution representations. 140 mammograms obtained from MIAS database was used in this study (Table 2).

Table 2. Number of training and testing set

\begin{tabular}{lcc}
\hline & \multicolumn{2}{c}{ database } \\
\multicolumn{1}{c}{ Category } & No. of training set & No. of testing set \\
\hline Normal & 36 & 18 \\
Anormal & 58 & 28 \\
\hline
\end{tabular}

\subsection{Feature Extraction}

Feature extraction involves simplifying the amount of resources required to describe a large set of data accurately. In the proposed method of the GIST descriptor built by Torralba [12], it is close enough to the Gabor filter bank. The new versions use the steerable pyramids. The "raw" descriptor is constructed as follows:

It turns the image into a bank of Gabor filters with $\mathrm{N} \sigma$ scales $\mathrm{N} \theta$ orientations scale, we obtain $\mathrm{N}=\mathrm{N} \theta \times \mathrm{N} \sigma$ images. Each image was divided into-M $\times \mathrm{M}$ sub-images. We calculate the energy of each sub image, so we get a vector of $\mathrm{N} \times \mathrm{M} \times \mathrm{M}$.and we use the conventional parameters:
1) $\mathrm{N} \sigma=4$
2) $\mathrm{N} \theta=8$
3) $M=4$

Then the vector size is 512 .

\subsection{Classification Phase}

In this phase, knn classifier is used to classify the images. The enhanced image is given as the input to classify mammograms into normal and abnormal. If it's contains tumor (microcalcification). Experiments were done with several values of $k$ and D0 value of cutoff needed for the homomorphic filter.

The performance of the proposed approaches for the classification of Normal and Anormal patterns is measured by classification accuracy, sensitivity and specificity.

It has been computed based on the number of correctly detected normal/abnormal images in order to evaluate the efficiency and robustness of the algorithm. The Metric is as follows:

$$
A(\%)=\frac{\text { Total number of correctly classified images }}{\text { Total number of images }}
$$

MIAS database is employed for experiments. 94 training images and 46 Testing Images are used.

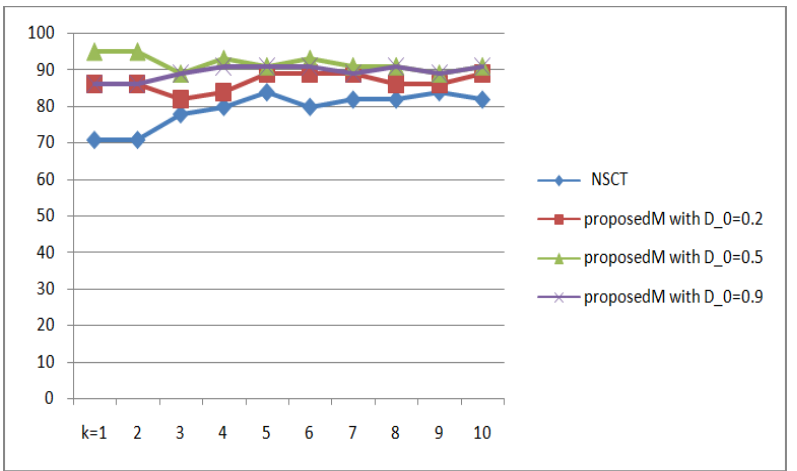

Figure 6. Classification accurancy rate the NSCT and proposed method with different value of cutoff needed for the homomorphic filter 
Figure 6 illustrates the classification accurancy rate obtained from the method used. From the figure are concluding that the proposed method is better than ather method in classification rate.

From the Figure 6 the best results from all the transforms (NSCT, proposedM with D_0 $=0.2, D \_0=0.5$ and $D \_0=0.9$ ) are always obtained for $k=1$. but for the transforms are individually producing their best results for different values of $k$.

The best results are obtained for $k=5$; where as for proposedM with $D_{-} 0=0.2$ the corresponding value of $k=5$ as for proposed Method (proposedM) with $D_{-} 0=0.9 \bar{K}=5$ as for proposedM with $\mathrm{D} \_0=0.5 k=1$ shows the best accurancy rate $95 \%$.

\section{Conclusion}

Contrast enhancement has an important role in Image processing as it extract the useful information from the distorted image. Image enhancement is used for improving the visual quality of an image.In this paper, we propose a hybrid the Contourlet and Hommomorphic Filtering for Enhancing Mammograms. The proposed method gives an impressive enhancement of the microcalcifications and an automatic classification for classifying the digital mammogram has been proposed. Preliminary experiments are carried out on MIAS database. From the experimental results, it is observed that the proposed mammogram classification system based on Gist feature with D_0 $=0.5$ set gives the better performance of $95 \%$ of classification rate.

\section{References}

[1] G Kom, A Tiedeu, M Kom, C Nguemgne, J Gonsu. Détection automatique des opacités dans les mammographies par la méthode de minimisation de la somme de l'inertie. ELSEVIER. 2005: 347356.

[2] http://www.contrelecancer.ma/documents/rapport-dactivites-2006/consulté le 12/04/2012

[3] Lure FYM, Jones PW, Gaborski RS. Multi-resolution unsharp masking technique for mammogram image enhancement. In SPIE Proceedings. 1996; 27(10): 830-839.

[4] S Helan, Jean-Christophe Burie, Thierry Bouwmans, Stéphane Bazeille. Object detection in underwater images. 2006.

[5] Wenli Liu, Peng He, Hui Li, Hongbo Yu. Improvement on the Algorithm of Homomorphic Filtering. International Conference on Electrical and Computer Engineering Advances in Biomedical Engineering. 2012; 11.

[6] MN Do, M Vetterli. The contourlet transform: an efficient directional multiresolution image representation. IEEE Trans.Image Proc. 2005; 14(12).

[7] Yue Lu, Minh N. A new contourlet transform with sharp frequency localization. Proc. of IEEE International Conference on Image Processing. 2006.

[8] PJ Burt, EH Adelson. The Laplacian pyramid as a compact image code. IEEE Trans. Comm. 1983; 31: $532-540$.

[9] RH Bamberger, MJT Smith. A filter bank for the directional decomposition of images: theory and design. IEEE Trans. Signal Proc. 1992; 40(4): 882-893.

[10] K Bovis, S Singh. Enhancement Technique Evaluation using Quantitative Measures on Digital Mammograms. Proceedings of 5th International Workshop on Digital Mammography. 2001; 547-553.

[11] Vikrant Bhateja, Swapna Devi. An Improved Non-Linear Transformation Function for Enhancement of Mammographic Breast Masses. 2011 3rd International Conference on Electronics Computer Technology (ICECT 2011). 2011.

[12] Aude Oliva, Antonio Torralba. Modeling the shape of the scene: a holistic representation of the spatial envelope. International Journal of Computer Vision. 2001; 42(3): 145-175. 